Linkspartei sich sehr kompromissbereit zeigte. Hinsichtlich einer verlässlichen und vertrauensvollen Zusammenarbeit mit dem Spitzenpersonal gab es seitens der SPD mit beiden potenziellen Partnern Probleme. Bei der CDU befürchtete man ein Wiederaufbrechen der alten Querelen, bei der Linkspartei war es die Stasi-Vergangenheit des linken Spitzenpersonals.

(8) Strategisch sprach für die SPD vieles für den Versuch, die Linke durch Einbindung in eine Koalition zu „entzaubern“ und damit gleichzeitig zu verhindern, dass sie sich als Oppositionspartei populistisch gegen die angesichts der Finanz- und Wirtschaftskrise notwendigen Sparmaßnahmen stellen und damit die SPD bei der nächsten Landtagswahl noch mehr gefährden würde. Nach mehreren Sondierungsgesprächen mit CDU und Linken nahmen die Sozialdemokraten Koalitionsverhandlungen mit der Linkspartei auf, was in Teilen beider Parteien auf Kritik stieß, letztlich jedoch mit großer Mehrheit gebilligt wurde.

(9) Matthias Platzeck wurde am 6. November 2009 vom Landtag erneut zum Ministerpräsidenten gewählt. Von den 86 anwesenden Abgeordneten stimmten 54 für ihn, 32 votierten gegen ihn, Enthaltungen gab es keine. Nach der Landessatzung ernannte Platzeck die Mitglieder der neuen Regierung. Die SPD erhielt fünf Ministerposten, die Linke vier.

(10) Schon kurz nach Aufnahme der Regierungsgeschäfte sorgten zwei neue Fälle von Stasi-Verstrickungen in der Fraktion der Linken dafür, dass Rot-Rot politisch in schwieriges Fahrwasser geriet.

\title{
Die schleswig-holsteinische Landtagswahl vom 27. September 2009: Ministerpräsident auf Abruf kann nach vorgezogener Neuwahl schwarz-gelbe Wunschkoalition bilden
}

\section{Patrick Horst}

Mit der Bildung der Großen Koalition im April 2005 war die CDU erstmals nach 17 Jahren wieder in der Regierung des nördlichsten deutschen Bundeslandes vertreten. Der Auftrag zur Regierungsbildung war Peter Harry Carstensen (CDU), der die Mehrheit für die angestrebte schwarz-gelbe Koalition am Wahlabend knapp verpasst hatte, unverhofft doch noch in den Schoß gefallen, nachdem es der seit 1993 regierenden Ministerpräsidentin Heide Simonis (SPD) zuvor in vier Wahlgängen nicht gelungen war, die absolute Mehrheit des Schleswig-Holsteinischen Landtages zur Unterstützung einer vom SSW tolerierten rotgrünen Koalition zu bringen. ${ }^{1}$ Das Scheitern von Rot-Grün in Schleswig-Holstein und kurz darauf auch in Nordrhein-Westfalen hatte Bundeskanzler Gerhard Schröder (SPD) veranlasst, die Wahl zum Deutschen Bundestag über den Weg einer „unechten“ Vertrauensfra-

1 Siehe zu den Turbulenzen der Regierungsbildung 2005 bei Thomas Saretzki / RalfTils, Die schleswig-holsteinische Landtagswahl vom 20. Februar 2005: Geheime Stimmverweigerung für Ministerpräsidentin Heide Simonis erzwingt Große Koalition, in: ZParl, 37. Jg. (2006), H. 1, S. 145 163, insbesondere S. $155-161$. 
ge $^{2}$ auf den September 2005 vorzuziehen. Bei dieser Wahl wurde Rot-Grün dann zwar auch im Bund abgewählt; wie in Schleswig-Holstein reichte es jedoch nicht zum ungefilterten Regierungswechsel, und so blieb nur die Große Koalition als einzig stabile Bündnisoption übrig. Die Kieler Koalitionsbildung im Frühjahr 2005 war das Produkt der Handlungsrestriktionen in einem Parteiensystem, das sich in der zweiten Amtszeit der rot-grünen Koalition stärker fragmentiert und polarisiert hatte sowie insgesamt fluider geworden war - eine Entwicklung, die sich nach der Bildung der Großen Koalition in Berlin im November 2005 fortsetzen sollte. ${ }^{3}$

\section{Die Ausgangslage}

Die aus der Not geborene Große Koalition in Kiel stand von Anfang an im Zeichen persönlicher Animositäten zwischen Ministerpräsident Peter Harry Carstensen und dem starken Mann der schleswig-holsteinischen SPD, Innenminister Ralf Stegner. Der über „theatralische Politik" promovierte Politologe ${ }^{4}$ brachte sich von Anfang an immer wieder mit Kritik am Koalitionskurs ins Gespräch, was den CDU-Ministerpräsidenten mehrmals dazu veranlasste, mit dem Bruch der Koalition zu drohen und den Rücktritt seines ehrgeizigen Innenministers zu fordern. Um die Fortführung der Koalition zu ermöglichen, kündigte Stegner, der im März 2007 auch Landesvorsitzender seiner Partei geworden war, schließlich im September 2007 an, von seinem Ministeramt zurückzutreten. SPD-Fraktionschef Lothar Hay rückte im Januar des folgenden Jahres auf den Posten des Innenministers, Parteichef Stegner übernahm von Hay dessen Amt als Fraktionsvorsitzender und ließ sich als Spitzenkandidat der SPD für die im Mai 2010 anstehende Landtagswahl nominieren. Die Personalrochade begründete Stegner auch damit, nun nicht mehr in die Kabinettsdisziplin eingebunden zu sein und somit freien Wahlkampf führen zu können. ${ }^{5}$

Für die Stabilität der Koalition bedeutete dies im weiteren Verlauf nichts Gutes. Stegner profilierte sich - auch bundespolitisch - als Fürsprecher einer Öffnung zur Linkspartei ${ }^{6}$

2 Zur Diskussion um „unechte“ und „echte“ Vertrauensfragen sowie zu Vorschlägen der Verfassungsreform in Richtung eines Selbstauflösungsrechts des Bundestages vgl. Karlheinz Niclauß, Auflösung oder Selbstauflösung? Anmerkungen zur Verfassungsdiskussion nach der Vertrauensfrage des Bundeskanzlers 2005, in: ZParl, 37. Jg. (2006), H. 1, S. 40 - 46; Heinrich Pehle, Verfassungspraxis im Zwielicht? Die Problematik „unechter Vertrauensfragen“ und „vorgezogener“ Bundestagswahlen, in: Eckhard Jesse / Roland Sturm (Hrsg.), Bilanz der Bundestagswahl 2005. Voraussetzungen, Ergebnisse, Folgen, Wiesbaden 2006, S. 177 - 187.

3 Vgl. Oskar Niedermayer, Das fluide Fünfparteiensystem nach der Bundestagswahl 2005, in: ders. (Hrsg.), Die Parteien nach der Bundestagswahl 2005, Wiesbaden 2008, S. 9 - 35; Eckhard Jesse, Parteiensystem im Wandel? Das deutsche Parteiensystem vor und nach der Bundestagswahl 2005, in: ders., Demokratie in Deutschland. Diagnosen und Analysen, herausgegeben von Uwe Backes I Alexander Gallus, Köln 2008, S. 294 - 316.

4 Ralf Stegner, Theatralische Politik made in USA. Das Präsidentenamt im Spannungsfeld von moderner Fernsehdemokratie und kommerzialisierter PR-Show, Münster 1992 (Dissertation an der Universität Hamburg 1992).

5 Vgl. Arne Boecker, Stegner tritt zurück - Koalitionsbruch abgewendet, SZ online, 17. September 2007.

6 Vor allem in der Diskussion um den Kurs der hessischen SPD nach der dortigen Landtagswahl im Januar 2008 trat Stegner als entschiedener Unterstützer der Spitzenkandidatin Andrea Ypsilanti hervor. Interessant hierzu die Deutungen bei Volker Zastrow, Die Vier. Eine Intrige, Berlin 2009, S. $96-101$. 
und setzte seine Kritik an der Koalition fort. Neben den Sparbeschlüssen der Landesregierung gab aus Stegners Sicht vor allem das Krisenmanagement der finanziell schwer angeschlagenen HSH Nordbank Grund zur Beanstandung. Auch Wirtschaftsminister Werner Marnette (CDU) hatte es im März 2009 als „absolut unprofessionell“ bezeichnet und zum Anlass seines Rücktritts genommen. ${ }^{7}$ Im Juli 2009 kam es zum persönlichen Zerwürfnis zwischen Carstensen und Stegner im Streit über eine umstrittene „Ausgleichszahlung“ von 2,9 Millionen Euro an Landesbank-Chef Dirk Jens Nonnenmacher. Dem Ministerpräsidenten kam der Koalitionskrach insofern gelegen, als er das Vertrauensverhältnis zu Stegner für endgültig zerrüttet erklären und Neuwahlen herbeiführen konnte. Da die Demoskopen Schwarz-Gelb im Juli 2009 einen deutlichen Sieg voraussagten ${ }^{8}$, verweigerte sich die SPD einer von Carstensen angestrebten Selbstauflösung des Landtags. Daraufhin entließ der Ministerpräsident, der mit einigen Stimmen aus der SPD-Fraktion zur Erreichung der Zweidrittelmehrheit gerechnet hatte, alle vier SPD-Minister und wählte den Weg einer „unechten Vertrauensfrage“, um noch parallel zur Bundestagswahl vorgezogene Neuwahlen stattfinden lassen zu können.?

Die Ausgangslage für die Landtagswahl am 27. September war für Carstensen und die von ihm gewünschte schwarz-gelbe Koalition aus mindestens fünf Gründen günstig: Erstens war die Grundannahme aller Theorien des „ökonomischen Wählens“, wonach es dem Wähler zuallererst um die Möglichkeit geht, eine Regierung mit wirtschaftspolitisch nicht zufriedenstellender Bilanz abzuwählen ${ }^{10}$, für diese Wahl außer Kraft gesetzt. Zwar sank die Zufriedenheit der Bürger mit der schwarz-roten Landesregierung von lange Zeit stabilen Werten nahe der 50-Prozent-Marke seit dem Herbst 2008 rapide ab und betrug im September 2009 nur noch 29 Prozent. ${ }^{11}$ Dieser Vertrauensverlust verlief zeitlich parallel zu den Turbulenzen der HSH Nordbank, die von den Landesregierungen in Hamburg und Schleswig-Holstein mit drei Milliarden Euro gestützt und mit noch einmal zehn Milliarden Euro an staatlichen Bürgschaften abgesichert worden war. ${ }^{12}$ Unter den Bedingungen einer Großen Koalition konnten die Wähler aber nicht einfach einer großen Regierungspartei das Vertrauen entziehen und es der Regierungspartei im Wartestand übertragen, denn beide Koalitionspartner waren für das Missmanagement der Landesbank verantwortlich. Somit war zweitens absehbar, dass von der Finanz- und Wirtschaftskrise in erster Linie die kleinen Parteien in der Opposition - FDP, Linkspartei, Grüne, SSW - und in zweiter Linie diejenigen Parteien mit den höheren wirtschaftspolitischen Kompetenzzuschreibungen profitie-

7 Vgl. RalfWiegand, Marnette tritt zurück: „Schlechtes Management“, SZ online, 29. März 2009.

8 Infratest dimap, LänderTREND Schleswig-Holstein, erhob unmittelbar nach dem Koalitionsbruch am 18. Juli 2009 folgende Werte in der „Sonntagsfrage“: CDU 36, SPD 24, FDP 15, Grüne 14, SSW 3 und Linkspartei 5 Prozent. Bei der „Direktwahlfrage“ lag Carstensen mit 51 Prozent deutlich vor Stegner mit 19 Prozent.

9 Vgl. Veit Medick / Philipp Wittrock, Männerfehde sprengt Kieler Krawall-Koalition, Spiegel online, 15. Juli 2009. Siehe auch die Landtagsdebatte vom 17. Juli 2009: Schleswig-Holsteinischer Landtag, Plenarprotokoll 16/119, S. 8840 - 8859.

10 Siehe die Klassiker des „Economic Voting“: V.O. Key, Jr., The Responsible Electorate. Rationality in Presidential Voting, 1936-1960, Cambridge 1966; Morris P. Fiorina, Retrospective Voting in American National Elections, New Haven 1981; Michael S. Lewis-Beck, Economics and Elections, Ann Arbor 1992.

11 Vgl. Infratest dimap, WahlREPORT Landtagswahl in Schleswig-Holstein 27. September 2009, Berlin 2009, S. 26.

12 Vgl. Ralf Wiegand, a.a.O. (Fn. 7). 
ren würden. Das aber waren trotz aller Unzufriedenheit mit Ministerpräsident Carstensens persönlicher Leistung in der Landesbankkrise CDU und FDP. Allerdings waren die Wähler mit Blick auf die HSH Nordbank so realistisch, keiner Partei die Lösung des Problems zuzutrauen. ${ }^{13}$ Drittens war zu erwarten, dass in der Krise die Wirtschaftsthemen (Arbeitsplatzsicherung, Ankurbelung der Wirtschaft, Schuldenabbau, Bankenmanagement) auch tatsächlich die Wahl entscheiden und andere politische Problemfelder (Atomausstieg, Umweltschutz, Gerechtigkeitsfragen, Familien- und Kinderpolitik), in denen SPD, Grüne und Linke als kompetent galten, in den Hintergrund drängen würden. Nur die Bildungspolitik schaffte es im Vorfeld der Wahl, in die Phalanx wirtschaftlicher Themen einzubrechen, die vom Wähler als wichtig eingestuft wurden. Aber auch hier lagen CDU und FDP mit SPD, Grünen und SSW fast gleichauf bei den Kompetenzwerten. ${ }^{14}$

Hinzu kam viertens, dass die Bundespolitik in diesem Wahlkampf der CDU und besonders der FDP Rückenwind versprach. Die Frage nach der bundespolitischen Überformung der Landtagswahlen hat sich inzwischen zu einem ausgesprochenen Modethema der deutschen Politikwissenschaft entwickelt; als Konsens der Forschung hat sich herausgeschält, dass es diesen Einfluss sicher gibt, es jedoch unklar ist, wie und unter welchen Bedingungen er wirkt. Überdies scheint er in manchen Bundesländern ausgeprägter zu sein als in anderen, wobei Schleswig-Holstein eher zu denjenigen Bundesländern zählt, in denen er sich stärker geltend macht als anderswo. ${ }^{15}$ Immer aber gilt, dass der bundespolitische Einfluss auf das Verhalten bei einer Landtagswahl kontextabhängig ist. Es gibt strukturelle Voraussetzungen und situationsspezifische Konstellationen, in denen bundespolitische Themen besonders stark auf individuelle Entscheidungen bei Landtagswahlen durchschlagen. Zu den strukturellen Voraussetzungen gehört in Schleswig-Holstein immer schon eine Sozialstruktur, in der die Kernanhängerschaft der beiden Volksparteien unterrepräsentiert ist und die folglich, verstärkt noch einmal nach der Barschel-Affäre, zu relativ hoher Volatilität im Wahlverhalten geführt hat. ${ }^{16}$ Situationsspezifisch wurde der bundespolitische Einfluss bei

13 Vgl. Infratest dimap, a.a.O. (Fn. 11), S. 27 f.

14 Vgl. ebenda, S. $24-27$.

15 So die bilanzierende Einschätzung von Kerstin Völkl / Kai-Uwe Schnapp / Everhard Holtmann / Oscar W. Gabriel in ihrer Einleitung zu dem von ihnen herausgegebenen Sammelband „Wähler und Landtagswahlen in Deutschland", Baden-Baden 2008, S. 9 - 36, Tabelle 2 auf S. 31. Die jüngere Diskussion um „(d)ie bundespolitische Durchdringung von Landtagswahlen“ wurde eröffnet durch den gleichnamigen Beitrag von Frank Decker / Julia von Blumenthal in der ZParl, 33. Jg. (2002), H. 1, S. 144 - 165; auf den Daniel Hough / Charlie Jeffery in der ZParl, 34. Jg. (2003), H. 1, S. 79 - 94 antworteten. Der oben zitierte Sammelband von Kerstin Völkl/ Kai-Uwe Schnapp / Everhard Holtmann / Oscar W. Gabriel ist die überarbeitete Version eines Themenschwerpunktes in Heft 3/2007 der ZParl. Zur Weiterführung der Diskussion siehe auch Simone Burkhart, Parteipolitikverflechtung. Über den Einfluss der Bundespolitik auf Landtagswahlentscheidungen von 1976 bis 2002, in: PVS, 46. Jg. (2005), H. 1, S. 14 - 38; sowie Frank Decker, Höhere Volatilität bei Landtagswahlen? Die Bedeutung bundespolitischer „Zwischenwahlen“, in: Eckhard Jesse / Roland Sturm (Hrsg.), a.a.O. (Fn. 2), S. 259 - 279.

16 Übereinstimmend Roberto Heinrich, Das Parteiensystem Schleswig-Holsteins, in: Uwe Jun / Melanie Haas / Oskar Niedermayer (Hrsg.), Parteien und Parteiensysteme in den deutschen Ländern, Wiesbaden 2008, S. 431 - 451, S. 443; Bettina Bonde I Immo von Homeyer, Wahlrecht, Wahlsystem und Wahlergebnisse in Schleswig-Holstein, in: Göttrik Wewer (Hrsg.), Demokratie in Schleswig-Holstein, Opladen 1998, S. 343 - 370, S. 353; Suzanne S. Schüttemeyer, SchleswigHolstein, in: Jürgen Hartmann (Hrsg.), Handbuch der deutschen Bundesländer, Frankfurt am Main 1997, S. $581-612$, S. 600 f. 
der Landtagswahl 2009 dadurch befördert, dass Bundes- und Landesregierung von derselben Partei geführt wurden ${ }^{17}$ und sogar dieselbe Koalitionskonstellation im Bund wie im Land anzutreffen war. Die positiven Meinungsumfragen für eine schwarz-gelbe Koalition im Bund kamen damit auch den schwarz-gelben Wahlkämpfern an der Kieler Förde zugute. Im Bund wie im Land war die Koalition von CDU (CSU) und FDP die mit Abstand am besten bewertete Koalition; diese Option wurde auch dadurch gestärkt, dass die Große Koalition nach vier Jahren im Bund wie in Kiel an Attraktivität stark eingebüßt hatte und nur noch einem Fünftel der schleswig-holsteinischen Wähler als wünschenswert galt. ${ }^{18}$ Dass Bundestags- und Landtagswahl am selben Tag stattfanden, beförderte den bundespolitischen Einfluss - allein schon über die zwangsläufig höhere Wahlbeteiligung - noch zusätzlich: Nicht zufällig spielte die Terminfrage im Kalkül des Ministerpräsidenten, die Landtagswahl vorzuziehen, eine zentrale Rolle.

Fünftens war die Ausgangslage für Schwarz-Gelb diesmal günstig, weil die SPD - in Kiel nicht anders als in Berlin - im Grunde über keine realistische Machtoption jenseits der Großen Koalition verfügte, deren Fortsetzung niemand, die SPD noch weniger als die CDU, anstrebte. Vom Juli bis in den September 2009 hinein pendelte die SPD bei der Sonntagsfrage im Bund wie in Schleswig-Holstein um Werte von 25 Prozent. Auf den ersten Blick liegt die Vermutung nahe, dass es sich bei der Krise der Kieler SPD ebenfalls um einen bundespolitischen Einfluss handeln könnte. Ein genauer Blick auf die Zahlen macht jedoch deutlich, dass die SPD im Bund schon kurz nach der Bundestagswahl 2002 unter die 30-Prozent-Marke fiel und seitdem nie mehr über 34 Prozent in der bundesweiten Sonntagsfrage erreichte. Die Kieler SPD dagegen zeigte sich bis zur Landtagswahl im Februar 2005 bemerkenswert krisenfest und stürzte erst nach dem „Heide-Mord“ im März 2005 in den Werten der landesweiten Sonntagsfrage um zehn Prozentpunkte ab. ${ }^{19}$ Die Krise der SPD in Kiel war und ist somit zu gewichtigen Teilen hausgemacht, wofür auch das wenig vorteilhafte Image ihres Landesvorsitzenden spricht. Im Profilvergleich mit Carstensen schnitt Stegner besonders bei den „weichen“ Charaktereigenschaften extrem schlecht ab. ${ }^{20}$ Wie fest das „Bad-Boy-Image“ Stegners saß, wurde unmittelbar nach dem Bruch der Koalition im Juli 2009 durch Carstensen deutlich, als impulsiv jeder vierte Wähler hauptsächlich Stegner für das Scheitern der Koalition verantwortlich machte. ${ }^{21}$

17 Die Wirkungskraft dieses Einflussfaktors hat Michael F. Meffert, Wenn Affären Wellen schlagen. Zum Einfluss der Landes- und Bundespolitik auf das Wahlverhalten in Schleswig-Holstein, in: Kerstin Völkl / Kai-Uwe Schnapp / Everhard Holtmann / Oscar W. Gabriel (Hrsg.), a.a.O. (Fn. 15), S. 287 - 308, für die Landtagswahlen in Schleswig-Holstein seit 1979 verifiziert.

18 Vgl. Infratest dimap, a.a.O. (Fn. 11), S. 39 (Erhebungszeitraum: 21. bis 23. September 2009). 47 Prozent hielten eine CDU/FDP-Koalition für sehr gut oder gut, 32 Prozent ein Bündnis aus CDU, FDP und SSW. Alle anderen sechs Varianten rangierten zwischen 20 und 25 Prozent.

19 Vgl. die Daten von Infratest dimap und Wahlrecht.de, http://www.infratest-dimap.de/umfragenanalysen/bundesweit/sonntagsfrage/, http://www.wahlrecht.de/umfragen/landtage/schleswig-holstein.htm (Abruf am 9. November 2009).

20 „Passt besser zu Schleswig-Holstein“: 61 zu 22 Prozent; „gibt bessere Figur in der Öffentlichkeit ab“: 69 zu 24; ,ist sympathischer“" 57 zu 22, vgl. Infratest dimap, a.a.O. (Fn. 11), S. 36 (Erhebung am 24. September 2009).

21 Nur jeder zehnte Wähler wies Carstensen die Hauptverantwortung zu, jeder zweite Wähler sah die Verantwortung auf beide Streithähne gleichermaßen verteilt. Zwei Monate später, als sich die Ereignisse schon etwas besser bewerten ließen, wurde Carstensen genauso häufig als Hauptschuldiger ausgemacht wie Stegner. Vgl. Infratest dimap, a.a.O. (Fn. 11), S. 37. 


\section{Der Wahlkampf}

Spätestens mit der Entlassung der vier SPD-Minister am 20. Juli 2009 hatte der Wahlkampf begonnen, den die SPD erstmals seit 21 Jahren wieder aus der Opposition heraus führen musste. Bereits am 1 . August verabschiedete sie auf einem außerordentlichen Parteitag in Lübeck ihr „Regierungsprogramm 2009 - 2014“. Unter der Überschrift „Das neue Jahrzehnt - Zukunft für Schleswig-Holstein“" stellte sie vor allem bildungs-, energie- und sozialpolitische Forderungen in den Mittelpunkt. Die SPD versprach kostenlose Bildung für alle von der Kindertagesstätte bis zum ersten Hochschulabschluss bis spätestens 2013 und setzte auf die Fortführung der Schulreform mit dem längeren gemeinsamen Lernen in der Gemeinschaftsschule. In der Energiepolitik garantierten die Sozialdemokraten den Ausstieg aus der Atomenergie (einschließlich der Abschaltung der Kernkraftwerke in Brunsbüttel und Krümmel), propagierten die konsequente Umstellung auf erneuerbare Energien bis 2020 und den Baustopp für neue Kohlekraftwerke. Einer Endlagerung von Kohlenstoffdioxid in Schleswig-Holstein erteilten sie eine Absage. Wie die Bildungspolitik richtete die Landes-SPD auch ihre Wirtschafts-, Arbeitsmarkt- und Gesundheitspolitik vor allem sozialpolitisch aus. Sie versprach „massive Rückendeckung für eine Bundespolitik, die Arbeitnehmerrechte achtet und ausbaut, den gesetzlichen Mindestlohn einführt und die solidarische Sozialversicherung schützt und stärkt"22. Zum Spitzenkandidaten nominierte die SPD ihren Partei- und Fraktionsvorsitzenden Ralf Stegner, der mit diesem Programm einen deutlichen Linkskurs einschlug. Zur Koalitionsfrage erklärte Stegner wie immer, dass er mit allen demokratischen Parteien spreche, dazu zählte für ihn auch die Linkspartei, und dass er von der „verbreiteten Ausschließeritis“ nichts halte. ${ }^{23}$

Der SPD folgten am Wochenende des 14. bis 16. August die drei kleinen Parteien auf dem politischen Spektrum links der Mitte mit ihren Wahlparteitagen: Grüne, SSW und Linkspartei. Die Grünen stellten ihr Wahlprogramm unter die Überschrift „Volle Kraft voraus! Gerade im Norden - Aus der Krise hilft nur Grün“. Für den Abbau des in der Finanzkrise aufgehäuften Schuldenberges und den Ausbau des Bildungssystems forderten sie, den Solidaritätsbeitrag mit dem Auslaufen der Förderung „Aufbau Ost“ beizubehalten. Die Grünen sprachen sich für die rückhaltlose Aufklärung des Missmanagements bei der HSH Nordbank aus, wozu sie den Parlamentarischen Untersuchungsausschuss in der neuen Wahlperiode fortsetzen wollten. Öffentliche Investitionen sollten nur noch „nachhaltig“ erfolgen - das heißt ausschließlich „in erneuerbare Energien, in Energieeinspar- und Effizienzprogramme und in Kultur und Bildung, von den Kindertagesstätten über die Schulen bis zur Förderung von Ausbildung und Hochschulen“. Die Grünen wollten die Umstellung auf erneuerbare Energien (besonders auf Windkraft) bereits zum Jahr 2015 erreichen; sie wollten die Gemeinschaftsschule zur „Schule der Zukunft“ machen, die Ganztagsschule ausbauen, die Kindertagesstätten als „Orte der frühkindlichen Bildung“ mittelfristig beitragsfrei stellen und die Unterfinanzierung der Hochschulen durch einen Hochschullastenausgleich zwischen den Bundesländern - und nicht durch Studiengebühren - beheben. Als

22 Das neue Jahrzehnt - Zukunft für Schleswig-Holstein. Regierungsprogramm 2009 - 2014 der SPD Schleswig-Holstein, beschlossen auf dem außerordentlichen Landesparteitag am 1. August 2009 in Lübeck, S. 7.

23 Zitiert bei Christian Teevs, Müntefering und Stegner beschwören Einigkeit der Genossen, Spiegel online, 31. Juli 2009. 
zentrales Wahlziel verkündeten sie die Verhinderung einer schwarz-gelben Koalition und die Bildung einer Regierung mit „klarer grüner Handschrift“. Sie zogen ohne Koalitionsaussage in den Wahlkampf; den neuen pragmatischen Kurs repräsentierten die Landtagsabgeordnete Monika Heinold auf Platz eins der Landesliste und der Parteivorsitzende Robert Habeck auf Platz zwei. Der bisherige Fraktionsvorsitzende Karl-Martin Hentschel, ein Befürworter von Rot-Grün, zog seine Landtagskandidatur angesichts des Kurs- und Generationswechsels in der Partei zurück. ${ }^{24}$

Ebenfalls ohne Koalitionsaussage zugunsten einer der beiden großen Parteien - aber mit dem klaren Willen, Regierungsverantwortung in einer Koalition zu übernehmen und somit vom bisherigen Tolerierungsmodell abzurücken ${ }^{25}$ - startete der SSW, angeführt von seinen beiden Landtagsabgeordneten Anke Spoorendonk und Lars Harms, in den Wahlkampf. Als „Partei der dänischen Minderheit und der Friesen“ betonte der von der Sperrklausel befreite, aber landesweit kandidierende Wählerverband auch 2009 wieder seinen regionalen Bezug, seine Bürgernähe und seine Orientierung ,an anerkannten gut funktionierenden skandinavischen Vorbildern" in der Sozial-, der Bildungs- und der Umweltpolitik. Mit seinen bildungspolitischen Forderungen zur Gemeinschaftsschule, zur Ganztagsschule, zur Reform der Lehrerausbildung und zur Kostenfreiheit der Bildung von der Kindertagesstätte bis zur Hochschule lag der SSW ganz dicht bei den Grünen und nicht allzu weit entfernt von der SPD; Gleiches galt für die Umweltpolitik. Mit ihren sozialpolitischen Vorschlägen zur aktiven Arbeitsmarktpolitik, zum Ausbau der Hartz IV-Leistungen und zur Abschaffung der Rente mit 67 positionierte sich die Minderheitenpartei sogar noch links von der SPD. ${ }^{26}$ Auf diesem Politikfeld wurde sie an Versprechungen nur noch von der Linkspartei übertroffen, die den Wählern in ihrem Aktionsprogramm eine staatlich finanzierte Rundumversorgung in Aussicht stellte: Schaffung von 70.000 Arbeitsplätzen, Umwandlung der Ein-Euro-Jobs in „reguläre Arbeitsplätze“, eine Schleswig-Holstein-Karte für Kinder, Ältere und sozial Schwache zur kostenlosen Nutzung von Bussen und Bahnen sowie von Sport-, Kultur- und Bildungseinrichtungen, eine flächendeckende Rücknahme von Privatisierungen, eine gebührenfreie Bildung von der - ganztägig anzubietenden - Kindertagesstätte bis zum Hochschulstudium und die Gemeinschaftsschule als alleinige Regelschule bis zum Ende des 10. Schuljahres. All diese Einzelversprechungen garnierte die Linke mit der übergeordneten Forderung, die Beseitigung der Armut als Staatsziel in die Landesverfassung aufzunehmen. Auf ihrem Spitzenplatz nominierte die Partei die frühere Landesvorstands-

24 Vgl. Volle Kraft voraus! Landtagswahlprogramm 2009 Bündnis 90/Die Grünen Schleswig-Holstein, beschlossen auf dem Landesparteitag in Neumünster vom 14. - 16. August 2009; Bodo Stade, Grüne mit neuer Doppelspitze - Karl-Martin Hentschel nicht mehr dabei, Kieler Nachrichten online, 15. August 2009.

25 Der SSW hatte aus den Ereignissen um die Regierungsbildung 2005 gelernt, als ihm von einigen Politikern der CDU und FDP in demokratiepolitisch skandalöser Weise das Recht abgesprochen wurde, Zünglein an der Waage zu sein, also sein demokratisch legitimiertes Mandat wahrzunehmen. Dazu Everhard Holtmann, Dürfen die das, wo die doch Dänen sind? Über den Umgang mit Macht und Minderheiten in Deutschland, in: ZParl, 36. Jg. (2005), H. 3, S. 616 - 629.

26 Vgl. Für uns im Norden. Näher dran - Wahlprogramm des SSW zur Landtagswahl am 27. September 2009, http://ssw.de/www/de/programme/landtagswahl/wahlprogramm2009/index.php (Abruf am 10. November 2009); Spoorendonk führt SSW in Landtagswahlkampf, Kieler Nachrichten online, 15. August 2009. 
sprecherin der Grünen Antje Jansen, die Regierungsbeteiligungen zunächst ausschloss und eine „knallharte Oppositionspolitik“ ankündigte. ${ }^{27}$

Am 22. August stellte die CDU ihr Wahlprogramm vor, das der Sanierung des Haushalts oberste Priorität einräumte: Bis zum Jahre 2020, dem Inkrafttreten des grundgesetzlich verankerten Neuverschuldungsverbots, sollte der Haushalt ausgeglichen sein. Während die Christdemokraten auf der Einnahmenseite keinen Handlungsspielraum jenseits einer wachstums- und beschäftigungsfreundlichen Wirtschaftspolitik ausmachten, identifizierten sie auf der Ausgabenseite Möglichkeiten zum Abbau von 4.800 Stellen im öffentlichen Dienst, auf den sie sich schon mit der SPD geeinigt hatten, zur Kürzung von Subventionen und zur Ausweitung Öffentlich-Privater-Partnerschaften. In der Bildungspolitik wollte die CDU alte ideologische Gräben nicht wieder aufreißen und stellte sich hinter die Schulreform der Großen Koalition, die bis Sommer 2010 zur Zweigliedrigkeit des Schulsystems führen sollte. Von der früher befürworteten Einführung von Studiengebühren rückte die CDU ab. In der Energiepolitik bekannte sie sich zum Ausbau der erneuerbaren Energien und zum Kampf gegen das im Bund diskutierte Kohlenstoffdioxid-Endlager in Nordfriesland. An Kohle- und Kernkraftwerken, für die sie Laufzeitenverlängerungen in Aussicht stellte, wollte die CDU vorerst festhalten, um eine bezahlbare Energieversorgung zu gewährleisten. In der Koalitionsfrage optierte sie eindeutig für die FDP als Wunschpartner, konnte sich aber auch die Grünen und den SSW als Partner vorstellen. Eine Koalition mit der SPD schloss Ministerpräsident Carstensen dagegen zumindest solange aus, wie diese von Ralf Stegner geführt würde. ${ }^{28}$

Die FDP mit Spitzenkandidat Wolfgang Kubicki ging noch weiter und schloss eine Koalition mit der SPD ,in den nächsten fünf Jahren definitiv“ aus. ${ }^{29}$ Die Liberalen gaben eine klare Koalitionsaussage zugunsten der CDU ab, stellten jedoch schon im Wahlkampf Bedingungen: Der seinerzeitige CDU-Finanzminister Rainer Wiegard dürfe nicht weiter Verantwortung für die HSH Nordbank tragen, und das Bildungsministerium müsse an die FDP gehen. Die Tür für die Grünen hielt Kubicki offen. Inhaltlich propagierte das Wahlprogramm der FDP eine konsequente Haushaltskonsolidierung, wozu der Verkauf der Landesanteile an der HSH Nordbank und an den Spielbanken zählte. Eine indirekte Haushaltssanierung wollte die FDP darüber hinaus durch die Freisetzung von Wachstumskräften über Mittelstandsförderung, Bürokratieabbau und diverse Infrastrukturprojekte erreichen. Besondere Kompetenz beanspruchten die Liberalen in der Bildungspolitik. Als einzige Partei in Schleswig-Holstein setzten sie sich für die Stärkung des Gymnasiums und den Erhalt der Realschule als Angebotsschule ein. In ihrer Forderung, die Schulen von „obrigkeitlicher Gängelung“ zu befreien und ihnen mehr Selbständigkeit einzuräumen, traf die FDP sich mit der CDU. In der Energiepolitik bekannten sich die Liberalen zum Atomausstieg. ${ }^{30}$

27 Vgl. Konsequent sozial! Aktionsprogramm für einen Politikwechsel in Schleswig-Holstein. Beschlossen vom Landesrat Die Linke.Schleswig-Holstein am 21. August 2009; Linkspartei will „knallharte Oppositionspolitik“ machen, Kieler Nachrichten online, 16. August 2009.

28 Vgl. CDU Schleswig-Holstein, Regierungsprogramm 2009 - 2014. Für unser Land, beschlossen vom CDU-Landesvorstand am 21. August 2009 in Ahrensburg; Uta Wilke, Schwarz-Gelb heißt das Ziel, Kieler Nachrichten online, 13. August 2009; dies., Energiemix oder Energiewende?, Kieler Nachrichten online, 21. September 2009.

29 Bodo Stade, Kubicki schließt Bündnis mit SPD aus, Kieler Nachrichten online, 28. August 2009.

30 Vgl. Landtagswahlprogramm 2009 - 2014 der FDP Schleswig-Holstein, beschlossen auf dem FDP Landesparteitag in Kiel am 6. September 2009; Bodo Stade, FDP beschließt Wahlprogramm: Kubickis Bedingungen, Kieler Nachrichten online, 7. September 2009. 
Die letzten Wochen vor der Wahl waren geprägt vom Straßen- und Veranstaltungswahlkampf sowie vom TV-Duell, das für den 23. September anberaumt worden war. Für den Herausforderer Stegner ging es in erster Linie um die Verbesserung seines Images. Sein Wahlkampfteam schickte ihn in die Fußgängerzonen, wo er Rosen und Werbematerial an die Passanten verteilte und sich betont locker gab. Seit Wochen schon existierten fast keine Fotos mehr mit seinem früheren Markenzeichen, der Fliege, sondern nur noch mit offenem Hemdkragen. Selbst in das Fernsehduell mit Carstensen ging Stegner ohne Halsbinder und mit dem erkennbaren Bemühen, stets zu lächeln. Damit er nicht immer wieder in seinen gewohnten Gesichtsausdruck mit notorisch heruntergezogenen Mundwinkeln zurückfiel, hatte er sich als Erinnerungsstütze einen Notizzettel mit einem Smiley auf das Pult gelegt. Auf Plakaten warb die SPD für ihren Spitzenkandidaten mit dem Slogan „sozialer. stärker. stegner." Dass Stegner - anders als sein Image eines unverbesserlichen Störenfrieds verhieß - auch ein Mannschaftsspieler sein kann, versuchte die SPD dadurch zu untermauern, dass sie ihn nach und nach Mitglieder seines „Zukunftsteams“ präsentieren ließ. Das Team hatte ausgesprochenes Lokalkolorit, Stegner lockte keine bundesweit bekannte (Politiker-) Persönlichkeit nach Kiel. ${ }^{31}$

Auch Ministerpräsident Carstensen führte einen Image-Wahlkampf, meinte dabei jedoch stärker als Stegner an positive Persönlichkeitswerte anknüpfen zu können. Mit Slogans wie „Peter Harry Carstensen. Seine Stärke ist seine Nähe“ oder „Ich kümmere mich: Arbeit sichern" wollte die CDU sich die volkstümliche Popularität des Landesvaters zunutze machen. Im Vergleich zu Stegner waren die hohen Sympathiewerte Carstensens im Vorfeld der Wahl zweifelsohne ein Plus für den Amtsinhaber; da sie nur relationale Werte widerspiegelten, blieb ihr absoluter Wert jedoch im Dunkeln. Außerdem war keineswegs ausgemacht, dass Carstensens landesväterliche Popularität vor dem Hintergrund erodierender Kompetenzwerte nicht zum Bumerang werden könnte. FDP-Wahlkämpfer Kubicki scheute sich jedenfalls nicht, dem Ministerpräsidenten gönnerhaft seine Affinität zu Volksfesten zuzugestehen und gleichzeitig „vernünftige“ Regierungsarbeit anzumahnen. Ganz falsch konnte er mit seiner Kritik an der mangelnden Kompetenz Carstensens (und Teilen seiner Regierungsmannschaft) nicht liegen, denn er stieg doch trotz allseits bekannter eigener Sympathiedefizite $^{32}$ zu demjenigen Politiker auf, mit dem die Wähler in Schleswig-Holstein am zufriedensten waren. ${ }^{33}$ Hier bestätigte sich wieder einmal die kommunikationswissenschaftliche Erkenntnis, dass beim Politikerimage Kompetenz und Führerqualitäten wichtiger sind als Sympathiewerte. ${ }^{34}$ Den Kandidaten von CDU und SPD musste deshalb zu denken geben, dass - mit Ausnahme vielleicht von Carstensens Führungskraft und Stegners sozialpolitischer Kompetenz - die harten Werte (Vertrautheit mit den Problemen der Bürger, Glaubwürdigkeit, wirtschaftspolitische Kompetenz) zu wünschen übrig ließen. Bemerkenswert war die

31 Vgl. Uta Wilke, Auf Wahlkampftour mit Ralf Stegner - Der Reiz der neuen Lockerheit, Kieler Nachrichten online, 14. September 2009; Stegner präsentiert 17-köpfiges „Zukunftsteam“, Kieler Nachrichten online, 18. September 2009.

$32 \mathrm{Zu} \mathrm{Kubicki} \mathrm{siehe} \mathrm{Jens} \mathrm{Schneider,} \mathrm{Herablassend,} \mathrm{eitel,} \mathrm{selbstbewusst} \mathrm{-} \mathrm{FDP-Chef} \mathrm{Kubicki} \mathrm{ist} \mathrm{in}$ Kiel sehr umstritten, Süddeutsche Zeitung online, 3. August 2009; Reinhard Bingener, Der Wolf im Wolfspelz. Das Aus von Schwarz-Rot in Kiel gibt Kubicki eine letzte Chance, in: FAZ vom 8. September 2009, S. 5.

33 Vgl. Infratest dimap, a.a.O. (Fn. 11), S. 33 (Erhebungszeitraum: 21. bis 23. September 2009).

34 Vgl. Frank Brettschneider, Spitzenkandidaten und Wahlerfolg. Personalisierung - Kompetenz Parteien. Ein internationaler Vergleich, Wiesbaden 2002, S. $154-220$. 
hohe Unzufriedenheit, die sowohl Carstensen als auch Stegner entgegenschlug und die beide zu einer Belastung für ihre Partei machte. ${ }^{35}$ Beide Kandidaten büßten im TV-Duell noch einmal an Zustimmung ein - insofern ist es gerechtfertigt, von zwei Verlierern des Fernsehduells zu sprechen. ${ }^{36}$

\section{Das Wahlergebnis}

Ähnlich wie im Jahre 2005 fiel das Wahlergebnis denkbar knapp aus: CDU und FDP erreichten zusammen mit 46,4 Prozent zwar einen etwas geringeren Zweitstimmenanteil als noch vor vier Jahren; dank der größeren Geschlossenheit ihrer Anhänger, wie sie sich vor allem im Stimmensplitting der FDP-Wähler ausdrückte, überquerte die schwarz-gelbe Koalition diesmal jedoch als Sieger die Ziellinie. 39 Prozent der FDP-Wähler gaben ihre Erststimme 2009 Kandidaten der CDU und verhalfen ihr so zum Gewinn von 34 Wahlkreisen - elf Sitze mehr, als ihr nach Proporz zustanden. Von den Grünen-Wählern dagegen gaben nur 27 Prozent ihre Erststimme einem SPD-Kandidaten, 2005 hatte dies noch jeder zweite getan. ${ }^{37}$ Auch dies trug dazu bei, dass die SPD diesmal nur sechs Wahlkreise gewann. Zusammen verfügen CDU (34) und FDP (15) über 49 von 95 Mandaten im deutlich vergrößerten Landtag, eine Stimme über der absoluten Mehrheit. Die SPD kam nur auf 25 Sitze, die Grünen gewannen zwölf, die Linken fünf und der SSW vier Sitze (vgl. Tabelle 1). (Am 22. Januar 2010 wurde das Wahlergebnis durch den Landeswahlausschuss korrigiert. ${ }^{38}$ ) Ihre absolute Mehrheit im Landtag hatte die schwarz-gelbe Koalition auch einer Unklarheit im schleswig-holsteinischen Landtagswahlrecht bezüglich des Ausgleichs der Überhangmandate zu verdanken. Weil nur acht der elf Mehrsitze der CDU ausgeglichen wurden, kehrten sich die Mehrheitsverhältnisse nach Verteilung der Ausgleichsmandate zugunsten von CDU und FDP um. ${ }^{39}$

Eindeutige Wahlsieger des 27. September waren die FDP und ihr Spitzenkandidat Kubicki. Gegenüber 2005 legten die Freien Demokraten um mehr als das Doppelte (8,3 Prozentpunkte) zu, erreichten nun 14,9 Prozent des Zweitstimmenanteils und 15 (korrigiert 14) Sitze im Landtag. Ihren Stimmenanteil verdoppeln konnten auch die Grünen, die 12,4 Prozent der Stimmen und zwölf Sitze gewannen. Erstmals in den Landtag schaffte es die Linkspartei, die den Stimmenanteil ihrer Vorgängerpartei PDS um 5,2 Punkte auf 6,0 Prozent ausbaute und fünf (korrigiert sechs) Sitze im Landtag errang. Auch der SSW gewann deutlich hinzu und erreichte 2009 4,3 Prozent der Stimmen, was ihm eine Verdoppelung seiner Mandate von zwei auf vier einbrachte. Die kleinen Parteien waren die Nutznie-

3559 beziehungsweise 58 Prozent waren weder weniger oder gar nicht zufrieden mit ihren Leistungen, vgl. Infratest dimap, a.a.O. (Fn. 11).

36 Vgl. ebenda, S. 36.

37 SSW- (18 Prozent) und Linkspartei-Wähler (13 Prozent) gaben in noch geringerem Umfang ihre Erststimme an einen Kandidaten der SPD, siehe Infratest dimap, a.a.O. (Fn. 11), S. 50.

38 Eine Nachzählung in einem Husumer Wahlkreis hatte zur Konsequenz, dass die FDP einen Sitz verlor und die Linke einen hinzugewann. Die schwarz-gelbe Koalition verfügt seither über kein Surplus-Mandat mehr. Vgl. Ulf B. Christen, Carstensens Mehrheit ist jetzt in Gefahr, in: Hamburger Abendblatt vom 23. Januar 2010, S. 22.

39 Vgl. zur Wahlsystemfrage den Beitrag von Eric Linhart und Harald Schoen in diesem Heft der ZParl. 
Tabelle 1: Ergebnisse der Landtagswahl in Schleswig-Holstein am 27. September 2009

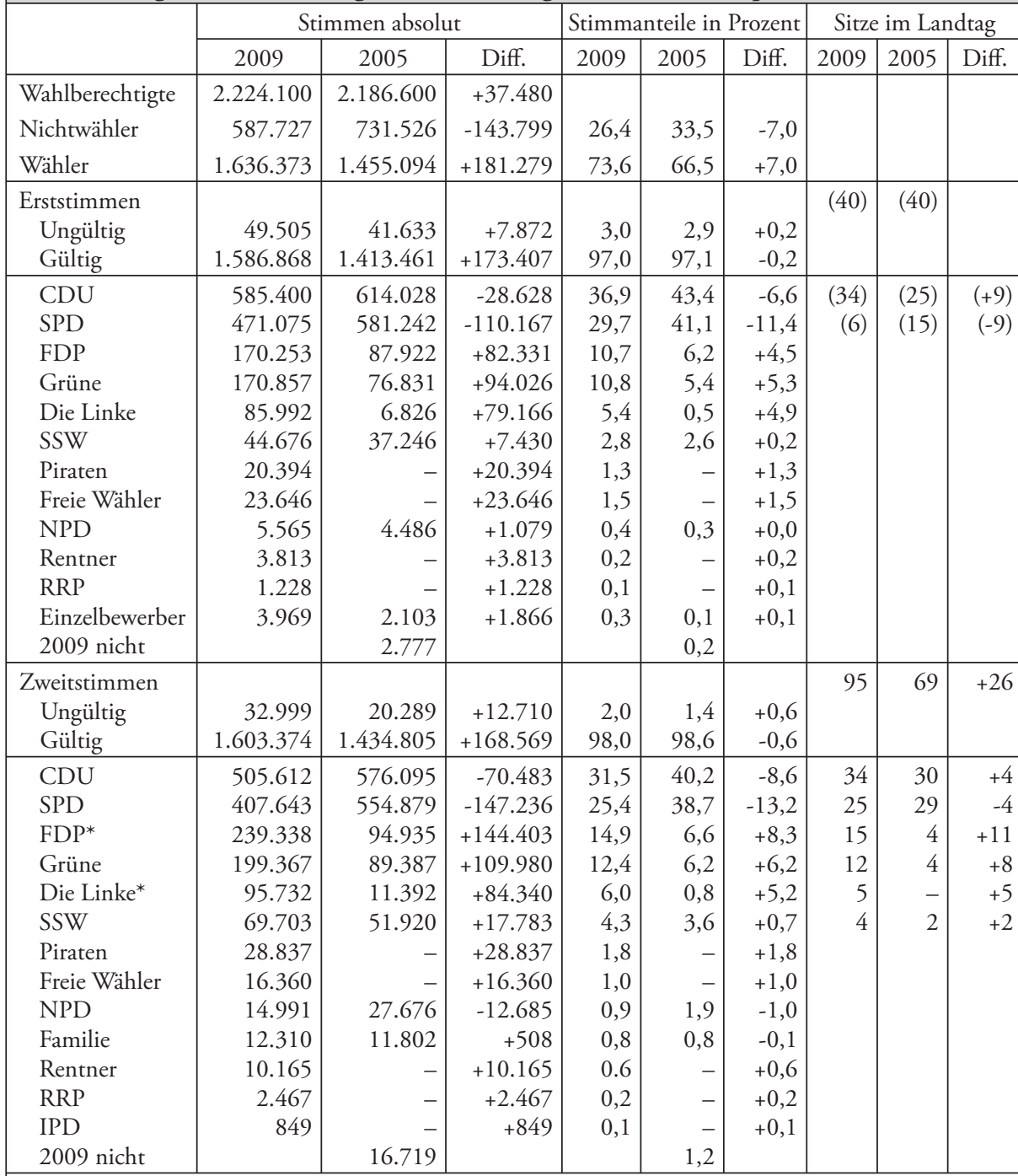

* Durch Beschluss des Landeswahlausschusses vom 22. Januar 2010 korrigiert auf: FDP 14, Die Linke 6 Sitze.

Quelle: Die Landeswahlleiterin des Landes Schleswig-Holstein, Endgültiges Ergebnis der Landtagswahl, Presseinformation vom 16. Oktober 2009.

ßer dieser vorgezogenen Neuwahl im Gefolge einer gescheiterten Großen Koalition, wie sich auch an den Erfolgen der Piratenpartei und der Freien Wähler Schleswig-Holstein ablesen lässt, die es beide über die Ein-Prozent-Schwelle brachten und Anspruch auf staatliche Parteienfinanzierung erwarben (vgl. Tabelle 1).

Wo die kleinen Parteien - mit Ausnahme der NPD, die mehr als die Hälfte ihres Stimmenanteils und den Anspruch auf staatliche Parteienfinanzierung verlor - durch die Bank zulegten, konnten die beiden großen Parteien nur verlieren. Die SPD büßte 13,2 Punkte 


\begin{tabular}{|l|l|c|c|c|c|c|}
\hline Tabelle 2: Vergleich der Landtagswahlergebnisse der beiden Volksparteien mit den Bundestags- \\
wahlergebnissen im Bund und in Schleswig-Holstein am 27. September 2009 \\
\hline & LTW in S-H 2009 & \multicolumn{2}{|c|}{ BTW in S-H 2009 } & BTW im Bund 2009 \\
\cline { 2 - 7 } & Stimmanteil & Diff. 2005 & Stimmanteil & Diff. 2005 & Stimmanteil & Diff. 2005 \\
\hline CDU (+CSU) & 31,5 & $-8,6$ & 32,2 & $-4,2$ & 33,8 & $-1,4$ \\
\hline SPD & 25,4 & $-13,2$ & 26,8 & $-11,4$ & 23,0 & $-11,2$ \\
\hline Quellen: Landeswahlleiterin Schleswig-Holstein; Bundeswahlleiter. \\
\hline
\end{tabular}

gegenüber 2005 ein und erreichte lediglich 25,4 Prozent der Wähler - ihr schlechtestes Landtagswahlergebnis in der Nachkriegsgeschichte Schleswig-Holsteins. Auch die CDU brachte mit einem Verlust von 8,6 Punkten gegenüber 2005 nur noch 31,5 Prozent der Wähler hinter sich. Nur 1950 hatte sie mit 19,8 Prozent schlechter abgeschnitten, was damals der Sondersituation des Flüchtlingszustroms und den exorbitanten Stimmenzuwächsen des GB/BHE geschuldet war. Selbst bei der Landtagswahl 1988 nach der BarschelAffäre hatte die CDU einen höheren Stimmenanteil erhalten. ${ }^{40}$ Während SPD-Kandidat Stegner noch auf die Ausnahmesituation der Großen Koalition im Bund und den für die Kieler SPD insofern ungünstigen bundespolitischen Gegenwind verweisen konnte, entfiel dieses Argument für den Ministerpräsidenten, der die Wahl schließlich nicht ganz uneigennützig auf den 27. September vorgezogen hatte. Wie die Analyse der Wählerströme zeigt, ging das Kalkül des Ministerpräsidenten auch auf: CDU und FDP profitierten überproportional von der durch die Terminzusammenlegung bewirkten hohen Wahlbeteiligung, die um sieben Punkte auf 73,6 Prozent gestiegen war. Beide Parteien mobilisierten deutlich mehr Stimmen aus dem Nichtwählerlager als alle anderen. ${ }^{41}$ Dennoch erzielte Carstensen ein schlechteres Ergebnis für seine Partei, als es diese in der Bundestagswahl in Schleswig-Holstein und im Bund erreichte. In dieser Hinsicht übertraf er sogar Stegner noch negativ. Daher erscheint die Vermutung plausibel, dass die Landespolitik zwar nicht das Bundestagswahlergebnis der Parteien „überformte“ - auch die kleinen Parteien in Schleswig-Holstein schnitten bei der Landtagswahl noch besser ab als bei der Bundestagswahl -, wohl aber den bundespolitischen Trend noch einmal verstärkte. ${ }^{42}$ Erst im Vergleich mit der Bundestagswahl wird deutlich, wie katastrophal das Abschneiden Peter Harry Carstensens, der seit dem Wahlabend als Ministerpräsident auf Abruf gelten muss, wirklich war (vgl. Tabelle 2).

\subsection{Motive der Wahl: Kandidaten, Kompetenzen, Kernwählerschaften}

Das Wahlergebnis der Landtagswahl 2009 war stärker kompetenzbasiert als noch vor vier Jahren: 56 Prozent der Wähler (2005: 41 Prozent) gaben an, dass ihre Entscheidung sich an den wahrgenommenen Parteikompetenzen orientierte, nur für jeweils 20 Prozent waren der

40 Zur Historie des Parteiensystems in Schleswig-Holstein vgl. Suzanne S. Schüttemeyer, a.a.O. (Fn. 16), S. $597-606$.

41 Vgl. Infratest dimap, a.a.O. (Fn. 11), S. 10.

42 Dass auch landespolitische Erfahrungen mit Parteien das Wahlverhalten im Bund beeinflussen, es also eine untrennbare Wechselwirkung zwischen bundes- und landespolitischer Ebene im bundesdeutschen Verbundföderalismus gibt, kommt bei der ganzen Diskussion um die bundespolitische Überformung der Landtagswahlen zu kurz. Vgl. die in Fußnote 15 zitierte Literatur. 
Kandidat oder die Parteibindung ausschlaggebend. CDU-Wähler orientierten sich etwas stärker am Kandidaten, 29 Prozent wählten die Partei aufgrund ihres Spitzenkandidaten. Für eine Partei, die den Ministerpräsidenten stellt, ist dies jedoch ein schwacher Wert und zeugt von der mangelnden Zugkraft Peter Harry Carstensens. Zum Vergleich: 2005 wählten 45 Prozent der SPD-Wähler ihre Partei wegen Heide Simonis, 2008 in Hamburg gaben sogar 55 Prozent der CDU-Wähler ihre Stimme der Partei dank Ole von Beust. ${ }^{43}$ Auch Ralf Stegner erwies sich als kein sonderlich zugkräftiger Kandidat, nur jeder fünfte SPD-Wähler entschied sich seinetwegen für die Sozialdemokraten. Beide Volksparteien, die CDU noch stärker als die SPD, litten unter der nachlassenden Bindung ihrer Kernwählerschaften. Nur noch 27 (CDU) beziehungsweise 26 Prozent (SPD) ihrer Anhänger wählten sie aus Treue. Damit standen die beiden Volksparteien zwar immer noch deutlich besser da als alle kleinen Parteien, die sich ihre Wähler zu zwei Dritteln (FDP, SSW) oder gar zu drei Vierteln (Grüne, Linkspartei) über ihre Kompetenzen verdienen mussten, aber erneut schlechter als vor vier Jahren. ${ }^{44}$

Wahlentscheidende Themen gab es 2009 viele - eine Folge des themenbezogenen Wahlkampfs und des breit aufgestellten Sechsparteiensystems in Schleswig-Holstein. Am häufigsten nannten die Wähler Wirtschaft (37 Prozent), Gerechtigkeit (29 Prozent), Arbeitsmarkt und Bildung (je 24 Prozent) sowie Umwelt/Klima (20 Prozent). CDU und FDP zogen die meisten Wähler mit ihrer wirtschafts- und arbeitsmarktpolitischen Kompetenz an, die FDP darüber hinaus mit ihrer steuer- und bildungspolitischen Profilierung. Gerechtigkeitsfragen spielten für Wähler der Linkspartei, der SPD und des SSW eine zentrale Rolle, während die Grünen mit ihrer umweltpolitischen Kompetenz die meisten Wähler an sich banden. Die Wirtschafts- und Finanzkrise sowie die von den Parteien vorgestellten Krisenrezepte waren ein wichtiges Thema - vor allem für die Wähler der CDU und der FDP -, aber keinesfalls das alles entscheidende, dem sich alle anderen Fragen unterordnen mussten. ${ }^{45}$

\subsection{Sozialstrukturelle und regionale Wahlergebnisse}

In sozialstruktureller und regionaler Hinsicht bestätigte die Landtagswahl in SchleswigHolstein altbekannte Zusammenhänge. Dennoch fällt auf, dass die beiden großen Parteien vor allem bei ihrer traditionellen Klientel überproportional verloren. Die CDU büßte besonders stark beim alten Mittelstand (Beamte, Selbstständige), bei Katholiken, Nichtmitgliedern von Gewerkschaften und insgesamt bei Männern ein. Genau bei diesen Gruppen gewann jedoch die FDP an Zuspruch, so dass diese klassischen Kernwählergruppen der CDU zumindest im „bürgerlichen Lager“ verblieben. Ähnlich verhielt es sich im „linken Lager", wo die Verluste der SPD bei ihren Stammwählern von Linkspartei und Grünen aufgefangen wurden. Die SPD verlor in den Berufsgruppen überdurchschnittlich bei Arbeitern, Angestellten, Arbeitslosen und Auszubildenden, wobei Arbeiter und Arbeitslose vornehmlich zur Linken, Angestellte zu den Grünen abwanderten. Die überproportionalen Verluste der SPD bei Gewerkschaftsmitgliedern waren der Konkurrenz der Linkspartei ge-

43 Vgl. Patrick Horst, Die Wahl zur Hamburger Bürgerschaft vom 24. Februar 2008: Wahlsieger Ole von Beust bildet die erste schwarz-grüne Koalition auf Landesebene, in: ZParl, 39. Jg. (2008), H. 3, S. $509-528$, S. 522.

44 Vgl. Infratest dimap, a.a.O. (Fn. 11), S. 48.

45 Vgl. ebenda, S. 49. 
schuldet, Konfessionslose wechselten in gleichem Maße zur Linken wie zu den Grünen. Darüber hinaus büßte die SPD stark an Zuspruch bei Frauen und in allen Altersgruppen unter 60 Jahren ein. Umgekehrt fand die SPD erhebliche Unterstützung in der Altersgruppe der über 60-Jährigen und bei Rentnern. In diesen beiden Gruppen wurde sie nur noch deutlich von der CDU übertroffen, was darauf hindeutet, dass die Volksparteien nach Jahrzehnten des unbegründeten Abgesangs nun tatsächlich in eine kritische Phase ihrer Existenz eingetreten sind. ${ }^{46}$

Die sozialstrukturellen Bestimmungsfaktoren des Wahlverhaltens lassen sich naturgemäß auch regional verorten. Die alte Arbeiterpartei SPD erzielte auch 2009 die besten Ergebnisse in ihren früheren Hochburgen, den industriellen Ballungszentren. Die sechs Wahlkreise, die sie direkt gewann, waren die drei Kieler und die drei Lübecker. Auch sonst schnitt die SPD in Städten wie Rendsburg und Neumünster oder in stadtnahen Gebieten des Hamburger Umlands wie Elmshorn, Pinneberg oder Lauenburg-Süd überdurchschnittlich ab, während sie in ländlichen Gebieten - vor allem Schleswigs - deutlich schlechtere Ergebnisse erhielt. In Schleswig, seinem Stammland, hatte der SSW wie immer seine Hochburg. Hier erzielte er mit 10,4 Prozent ein weit überproportionales Resultat; in den beiden Flensburger Wahlkreisen votierten 18,4 (Stadt) und 16,4 (Land) Prozent der Wähler für ihn. Im Landesteil Holstein kam der SSW dagegen nur auf 2,4 Prozent. Die CDU, Partei der Selbständigen und Landwirte, schnitt in den ländlichen Gebieten - in Schleswig wie im Westen Holsteins - besser ab als im Durchschnitt; auch im Hamburger Umland erzielte sie starke Ergebnisse. Den Wahlkreis Husum-Land in Nordfriesland gewann Peter Harry Carstensen ungefährdet. In den Städten, vor allem in Kiel und Lübeck, waren die CDU dagegen wie auch die FDP am schwächsten. Dort lässt sich der Gegensatz zwischen einem „bürgerlichen“ und einem „linken Lager“ nach wie vor am ehesten verorten: Neben der SPD erzielten hier auch Grüne und Linkspartei ihre besten Ergebnisse. ${ }^{47}$

\section{Formierung der Opposition und Regierungsbildung}

Nach der Wahl hatten es alle Fraktionen eilig, ihre Fraktionsvorstände für den neugewählten Landtag zu installieren. Besonders die Spitzenkandidaten der beiden großen Fraktionen wollten nach ihrer Wahlniederlage jeglichen Unmut im Keim ersticken und ihre Position in der Fraktion festigen. Dies gelang ihnen erstaunlich gut: Bereits am 29. September wählte die SPD-Fraktion Stegner mit 20 von 25 Stimmen zum Fraktionsvorsitzenden und somit neuen Oppositionsführer. Versuche innerhalb der SPD, noch für den November einen Sonderparteitag zur Analyse der Wahl einzuberufen, wurden von Stegner ebenso erfolgreich abgewehrt wie Vorschläge, den Partei- und Fraktionsvorsitz künftig zu trennen. Die CDU wählte am selben Tag den bisherigen Umweltminister Christian von Boetticher mit 28 von

46 Vgl. ebenda, S. 45 - 47. Zur Volksparteiendiskussion vgl. zum Beispiel jüngst Franz Walter, Im Herbst der Volksparteien? Eine kleine Geschichte von Aufstieg und Rückgang politischer Massenintegration, Bielefeld 2009. Wie kritisch es um die Volksparteien steht, verdeutlicht vielleicht mehr als alles andere die Tatsache, dass sich mittlerweile auch frühere Kritiker (die sich daran gar nicht mehr zu erinnern scheinen) berufen fühlen, zu ihrer Verteidigung ganze Bücher zu schreiben. Siehe Elmar Wiesendahl, Mitgliederparteien am Ende? Kritik der Niedergangsdiskussion, Wiesbaden 2006. Diese lebensrettende Maßnahme kommt jedoch, so ist zu befürchten, zu spät.

47 Vgl. Infratest dimap, a.a.O. (Fn. 11), S. $55-57$. 
34 Stimmen zum Fraktionsvorsitzenden. Er galt als Favorit Carstensens und wird in Kiel als „Kronprinz“ gehandelt, der den Ministerpräsidenten im Laufe der Wahlperiode ablösen könnte. Bei den vier „kleinen“ Parteien im Kieler Landtag gab es kaum Überraschungen: Die FDP bestätigte Fraktionschef Kubicki einstimmig im Amt. Ebenfalls einstimmig wählten die Grünen Spitzenkandidatin Monika Heinold zur Fraktionsgeschäftsführerin, während Robert Habeck als künftiger Fraktionsvorsitzender elf von zwölf Stimmen erhielt. ${ }^{48}$ Der SSW, der mit vier Mandaten seit 1954 erstmals wieder Fraktionsstatus erhalten hatte, wählte erneut Anke Spoorendonk zur Vorsitzenden. Die fünfköpfige Linksfraktion wird von Heinz-Werner Jezewski und Ellen Streitbörger gemeinsam geführt. ${ }^{49}$

Nach einer kurzen Verschnaufpause nach dem Wahlkampf und ersten Sondierungen zwischen Carstensen und Kubicki starteten die eigentlichen Koalitionsverhandlungen am Donnerstag, den 8. Oktober. Geleitet wurden die Verhandlungen von einer fünfköpfigen Steuerungsgruppe mit den Partei- (Carstensen und Jürgen Koppelin von der FDP) und Fraktionsvorsitzenden (von Boetticher und Kubicki) sowie Finanzminister Rainer Wiegard. Die Detailarbeit leisteten sechs Arbeitsgruppen, die zunächst einer großen Verhandlungsgruppe mit zwölf Vertretern der CDU und sieben der FDP berichteten, bevor etwaige Streitfälle wieder der kleinen Verhandlungsgruppe vorgelegt wurden. Die größten inhaltlichen Differenzen zwischen den Partnern waren in der Finanz-, der Bildungs- sowie der Innenund Rechtspolitik auszuräumen. Größtenteils setzte sich die FDP durch: Im öffentlichen Dienst sollen bis zum Jahre 2020 5.600 Stellen abgebaut werden; die über die Wahlkampfforderung der CDU noch hinausgehende Zielvorgabe wurde auf Wunsch der FDP mit einem Prüfauftrag an eine Haushaltsstrukturkommission versehen. Die Realschule bleibt unter der Voraussetzung, dass ein anhängiges Volksbegehren erfolgreich ist, als „Angebotsschule“ erhalten; die Gymnasien dürfen, wie von der FDP gefordert, selbst entscheiden, ob sie in acht oder neun Jahren zum Abitur führen. Der Schul-TÜV („EVIT“) wird abgeschafft. Im Polizeigesetz des Landes sollen von der Großen Koalition eingeführte Verschärfungen wie die Rasterfahndung und die Vorratsdatenspeicherung wieder rückgängig gemacht werden. ${ }^{50}$

Weitestgehend durchsetzen konnte die FDP auch ihren Machtanspruch bei der Verteilung und Zuschneidung der Ressorts. Anstatt der zwei von CDU-Fraktionschef Boetticher zugestandenen Ministerien erhielten die Liberalen nach einem effektiv inszenierten „Theaterdonner" die geforderten drei: das Sozial-, das Justiz und das Bildungsministerium (vgl. Tabelle 3). Außerdem wurde das Sozialministerium um den Bereich Arbeit erweitert, das Justizministerium erhielt die Zuständigkeit für die Atomaufsicht, dem Bildungsministerium wurde aus der Staatskanzlei der Aufgabenbereich Kultur zugeschlagen. Forderungen der FDP wurde auch mit zwei Machtbeschneidungen von CDU-Amtsträgern entsprochen: Finanzminister Wiegard musste die Aufsicht über die HSH Nordbank abgeben und Staatskanzleichef Heinz Maurus seinen Posten räumen. ${ }^{51}$ Die CDU konnte sich nur mit ihrem

48 Vgl. Boetticher und Stegner zu Fraktionschefs gewählt, Kieler Nachrichten online, 29. September 2009.

49 Vgl. Bodo Stade, Selbstbewusstes Kleeblatt, Kieler Nachrichten online, 19. Oktober 2009; „Bunter Haufen" wird äußerst rot, ebenda, 21. Oktober 2009.

50 Vgl. Schleswig-Holstein: Zuversicht nach Gesprächen über Schwarz-Gelb, Welt online, 8. Oktober 2009; Koalition des Aufbruchs, Koalitionsvertrag zwischen der CDU und der FDP in Schleswig-Holstein für die 17. Legislaturperiode des Schleswig-Holsteinischen Landtages, Kiel 2009.

51 Vgl. Claudia Ehrenstein, CDU und FDP haben es nach Streit jetzt eilig, Welt online, 14. Oktober 2009. 


\begin{tabular}{|l|l|}
\hline Tabelle 3: Das schleswig-holsteinische Kabinett (Stand: 27. Oktober 2009) \\
\hline Ministerpräsident & Peter Harry Carstensen (62, CDU) \\
\hline $\begin{array}{l}\text { Minister für Arbeit, Soziales und Gesundheit, } \\
\text { stellvertretender Ministerpräsident }\end{array}$ & Dr. Heiner Garg (43, FDP) \\
\hline Minister für Justiz, Gleichstellung und Integration & Emil Schmalfuß (63, parteilos) \\
\hline Minister für Bildung- und Kultur & Dr. Ekkehard Klug (53, FDP) \\
\hline Innenminister & Klaus Schlie (55, CDU) \\
\hline Ministerin für Landwirtschaft, Umwelt und ländliche Räume & Dr. Juliane Rumpf (53, CDU) \\
\hline Finanzminister & Rainer Wiegard (60, CDU) \\
\hline Minister für Wissenschaft, Wirtschaft und Verkehr & Jost de Jager (44, CDU) \\
\hline $\begin{array}{l}\text { Quelle: http://www.schleswig-holstein.de/Portal/DE/LandesregierungMinisterien/Landesregierung/Lan- } \\
\text { desregierung_node.html (Abruf am 3. November 2009). }\end{array}$ \\
\hline
\end{tabular}

Wunsch nach der Besetzung des Wirtschaftsressorts und Kompensationsgeschäften unterhalb der Ministerebene durchsetzen: Maurus wurde als neuer Europa-Staatssekretär und Bevollmächtigter Schleswig-Holsteins beim Bund in den Rang eines Kabinettsmitglieds erhoben. Die CDU erhielt das Vorschlagsrecht für immerhin sieben der zehn Staatssekretäre, darüber hinaus durfte sie einen Beauftragten für Integrationsfragen im FDP-geführten Justizministerium und einen Beauftragten für den Mittelstand im Wirtschaftsministerium benennen. ${ }^{52}$ Die Verhandlungserfolge der FDP waren auf drei Faktoren zurückzuführen: den infolge des Wahlergebnisses auf 30 Prozent angewachsenen Mandatsanteil der FDP an der Koalitionsmehrheit, die zentrale Rolle der FDP im Kieler „Koalitionsspiel“ und das Verhandlungsgeschick Kubickis im Verein mit der Ergebnisorientierung Carstensens. ${ }^{53}$

Dem angeschlagenen Ministerpräsidenten war, das war Kubicki nicht entgangen, in erster Linie an einer schnellen Regierungsbildung gelegen. Die bekam er, aber zu dem von Kubicki diktierten Preis. Nach nur einer Woche waren die Koalitionsverhandlungen abgeschlossen. Bis auf den von der FDP zu nominierenden Justizminister und das Landwirtschaftsressort, das die CDU aus Quotengründen an eine Frau vergeben musste, waren alle Ministerämter besetzt (vgl. Tabelle 3). Tags darauf stimmten die Landesvorstände der Parteien und die Fraktionen den Vereinbarungen zu; am Samstag, den 17. Oktober, konnte der Koalitionsvertrag unterzeichnet werden. Eine Woche später, am 24. Oktober, nahmen der Landesparteitag der CDU in Husum und der Landeshauptausschuss der FDP in Kiel den Koalitionsvertrag an. Auf dem CDU-Parteitag kam es zu vernehmbarer Kritik an der Verhandlungsführung Carstensens und den nominierten Ministern Klaus Schlie und Rainer Wiegard. Das Festhalten an Wiegard könnte ein strategischer Schachzug der CDU-Fraktion und ihres Vorsitzenden Boetticher gewesen sein, die sich Wiegards Entlassung für den Fall weiterer Enthüllungen des wieder eingesetzten Parlamentarischen Untersuchungsausschusses zur HSH Nordbank ,aufheben“ wollen. Im Falle eines möglichen Stabswechsels im

52 Koalition des Aufbruchs, a.a.O. (Fn. 49), S. 56 - 57.

53 Kubicki machte die keineswegs allzu abwegige Rechnung auf, dass der Ministerpräsident wie zwei Minister zähle, somit neun Posten zu vergeben wären, von denen der FDP entsprechend ihrem Mandatsanteil drei zustehen würden. Auch hatte der Fraktionsvorsitzende der FDP richtig erkannt, dass die CDU über keine andere Koalitionsoption verfügte. Vgl. Peter Höver, Kubicki: Union hat zur FDP keine Alternative, in: Schleswig-Holsteinischer Zeitungsverlag online, 5. Oktober 2009. 
Ministerpräsidentenamt zur Mitte der Wahlperiode würden sich für Boetticher Spielräume für ein Kabinettsrevirement eröffnen. ${ }^{54}$

Am 27. Oktober, 30 Tage nach der Landtagswahl, erfolgte die Wahl Peter Harry Carstensens zum Ministerpräsidenten im Schleswig-Holsteinischen Landtag mit 50 gegen 45 Stimmen. ${ }^{55}$ Mindestens ein Abgeordneter der Oppositionsfraktionen hatte für Carstensen gestimmt. Mit der Großen Regierungserklärung am 18. November und der anschließenden Generaldebatte war die Regierungsbildung förmlich abgeschlossen. ${ }^{56}$

\section{Zusammenfassung}

(1) CDU und FDP erreichten zusammen zwar einen etwas geringeren Zweitstimmenanteil als noch vor vier Jahren; dank der größeren Geschlossenheit ihrer Anhänger, wie sie sich vor allem im Stimmensplitting der FDP-Wähler ausdrückte, überquerte die schwarz-gelbe Koalition, die sich faktisch schon im Wahlkampf formiert hatte, diesmal jedoch als Sieger die Ziellinie. Aufgrund eines - rechtlich umstrittenen - begrenzten Mehrsitzausgleichs verfügt die schwarz-gelbe Koalition über eine absolute Mehrheit von 49, seit Ende Januar 2010 nur noch 48 der 95 Sitze im deutlich vergrößerten Landtag.

(2) Eindeutiger Wahlsieger des 27. September war die FDP, die gegenüber 2005 um mehr als das Doppelte auf knapp 15 Prozent zulegte und 15 (korrigiert 14) Sitze im Landtag erreichte. Ihren Stimmenanteil verdoppeln konnten auch die Grünen, die über zwölf Prozent und zwölf Sitze gewannen. Erstmals in den Landtag schaffte es die Linkspartei mit sechs Prozent und fünf (korrigiert sechs) Mandaten. Der SSW erzielte über vier Prozent und vier Sitze. Die kleinen Parteien waren die Nutznießer dieser vorgezogenen Neuwahl im Gefolge einer gescheiterten Großen Koalition. Schleswig-Holstein verfügt nun über ein stark fragmentiertes Sechsparteiensystem, „effektiv“ sind es knapp fünf. Mit der Piratenpartei und den Freien Wählern erwarben zwei weitere Kleinparteien Anspruch auf staatliche Teilfinanzierung.

(3) Verlierer der Wahl waren die Parteien der Großen Koalition: Die SPD büßte über 13 Punkte gegenüber 2005 ein und erreichte nur noch ein Viertel der Wähler - ihr schlechtestes Landtagswahlergebnis in Schleswig-Holstein. Auch die CDU brachte mit einem Verlust von knapp neun Punkten nicht einmal mehr ein Drittel der Wähler hinter sich. Nur 1950 hatte sie noch schlechter abgeschnitten.

(4) Eine bundespolitische Formung der Landtagswahl in Schleswig-Holstein hat es durch die konformen Koalitions- und Wahlkampfmuster gegeben, eine „Überformung“ jedoch nicht. CDU und FDP profitierten zwar vom bundespolitischen Rückenwind, dennoch hatten die ausgeprägte Schwäche von CDU und SPD wie die Stärke der FDP in Schleswig-Holstein auch Gründe, die eindeutig in der Landespolitik zu finden waren. Das vergleichsweise schwache Abschneiden der Linkspartei im Norden hatte eben-

54 Unbelegte Spekulation des Verfassers, die anknüpfen kann an: Ulf B. Christen, Koalitionsverhandlungen in Kiel. Auf die Plätze: Wettlauf um Ministerposten, in: Hamburger Abendblatt vom 9. Oktober 2009, S. 24.

55 Vgl. Schleswig-Holsteinischer Landtag, Plenarprotokoll 17/1, 27. Oktober 2009, S. 13.

56 Vgl. ebenda, Plenarprotokoll 17/2, 18. November 2009. 
falls regionale Ursachen: eine zerstrittene Landesorganisation und den dezidierten Linkskurs Stegners. Der SSW ist an sich schon eine regionale Besonderheit.

(5) Das Vorziehen der Landtagswahl auf den Termin der Bundestagswahl war für den Anstieg der Wahlbeteiligung um sieben Punkte auf 73,6 Prozent verantwortlich. Das Kalkül von Carstensen ging auf: CDU und FDP profitierten von der Zusammenlegung der Wahltermine stärker als alle anderen Parteien und mobilisierten die meisten Nichtwähler.

(6) Mit Peter Harry Carstensen und Ralf Stegner waren die hauptverantwortlichen Akteure für das Scheitern der Großen Koalition als Spitzenkandidaten ihrer Parteien angetreten. Obwohl sie beide die Wähler nicht zu überzeugen wussten, hielten sie sich mangels innerparteilicher Alternativen vorerst in ihren Ämtern. Stegner scheint derzeit keinen Konkurrenten in der SPD fürchten zu müssen. Carstensen dürfte aber ein Ministerpräsident auf Abruf sein - mit Fraktionschef Christian von Boetticher steht ein „Kronprinz“ bereit, der ihn im Laufe der Wahlperiode ablösen könnte.

(7) In den zügig geführten Koalitionsverhandlungen setzte sich die FDP auf breiter Front durch: In der Finanz-, der Bildungs- sowie der Innen- und Rechtspolitik trägt der Koalitionsvertrag vorrangig die Handschrift der Liberalen. Auch bei der Verteilung und beim Zuschnitt der Ressorts schnitt die FDP etwas besser ab, als es der reinen Arithmetik entsprochen hätte. Der Erfolg der Freien Demokraten bei den Koalitionsverhandlungen ist ihrem überwältigenden Wahlsieg geschuldet, ihrer Rolle als zentraler "Spieler" im Koalitionspoker und dem Verhandlungsgeschick ihres Fraktionsvorsitzenden, der die Schwäche des angeschlagenen Ministerpräsidenten geschickt auszunutzen wusste.

(8) Die selbsterklärte „Koalition des Aufbruchs“ sieht einer ungewissen Zukunft entgegen. Trotz der zügigen Regierungsbildung ist ihre Stabilität keineswegs gesichert. Die Schnelligkeit war teils der Schwäche der CDU und ihres angeschlagenen Ministerpräsidenten geschuldet, teils auch deren mangelnder Konfliktbereitschaft. Konflikte mit dem selbstbewussten kleinen Koalitionspartner können somit prognostiziert werden zumal die Koalition vor allem finanzpolitisch schwierige Entscheidungen vertagt hat. 